\title{
A NEW TYPE OF VECTOR FIELD AND INVARIANT DIFFERENTIAL SYSTEMS
}

\author{
H. H. JOHNSON ${ }^{1}$
}

In [1] Robert Hermann introduced the concept of tangent vector fields on the space of maps of one manifold into another. A special type of these are the " $k$-vector fields" which were studied in [3], where this author defined their bracket and exponential. This paper explores further the analogy with classical continuous groups. Specifically, we study invariance of systems of partial differential equations under $k$-vector fields.

1. Introduction. Every map and manifold is $C^{\infty}$ unless otherwise noted. $J^{k}=J^{k}(N, M)$ is the manifold of $k$-jets $j_{x}^{k}(f)$ of order $k$ of maps $f: N \rightarrow M$ from the manifold $N$ to the manifold $M . \alpha$ and $\beta$ are the source and target projections, $\rho_{i}^{\boldsymbol{k}+\boldsymbol{i}}: J^{k+i} \rightarrow J^{\boldsymbol{i}}$ the usual projection. $T(M)$ denotes the tangent bundle to $M, M_{y}$ the tangent space at $y \in M, \pi$ the tangent bundle projection. $C^{\infty}(Q)$ is the algebra (over the reals $R$ ) of $C^{\infty}$ real-valued functions on the manifold $Q$.

A $k$-vector field is a map $\theta: C^{\infty}(M) \rightarrow C^{\infty}\left(J^{k}\right)$ which is linear over $R$ and satisfies

$$
\theta(F G)=(F \circ \beta) \theta(G)+(G \circ \beta) \theta(F) .
$$

In [3] the $i$ th prolongation $P^{i} \theta: C^{\infty}\left(J^{i}\right) \rightarrow C^{\infty}\left(J^{i+k}\right)$ was defined. This satisfies, for $H \in C^{\infty}(M), F$ and $G \in C^{\infty}\left(J^{i}\right), P^{i} \theta(F G)=\left(F \circ \rho_{i}^{\boldsymbol{k}+\boldsymbol{i}}\right) P^{i} \theta(G)$ $+\left(G \circ \rho_{i}^{k+i}\right) P^{i} \theta(F)$ and $P^{i} \theta(H \circ \beta)=\theta(H) \circ \rho_{i}^{k+i}$. Using these facts one sees that if $\theta$ and $\psi$ are $k$ - and $i$-vector fields, respectively, then $[\theta, \psi]=P^{i} \theta \circ \psi-P^{k} \psi \circ \theta$ is a $(k+i)$-vector field.

In local coordinates $\left(x^{i}\right)$ on $N,\left(y^{\lambda}\right)$ on $M,\left(x^{i}, y^{\lambda}, p_{j_{1}}^{\lambda}, \cdots, p_{j_{1}}^{\lambda} \cdots j_{k}\right)$ on $J^{k}$, where $i, j_{1}, \cdots, j_{k}=1, \cdots, n ; \lambda=1, \cdots, m$, we follow Kuranishi [4] in defining for each $F \in C^{\infty}\left(J^{k}\right), \partial_{j}^{f} F \in C^{\infty}\left(J^{k+1}\right)$ by

$$
\partial_{j}^{*} F=\frac{\partial F}{\partial x^{j}}+\frac{\partial F}{\partial y^{\lambda}} p_{j}^{\lambda}+\cdots+\frac{\partial F}{\partial p_{j_{1} \cdots j_{k}}^{\lambda}} p_{j_{1} \cdots j_{k} j}^{\lambda} .
$$

If $\theta$ is a $k$-vector field, then in local coordinates $\theta$ may be expressed in the form $\theta=a^{\lambda}\left(\partial / \partial y^{\lambda}\right)$ where the $a^{\lambda}$ are real-valued functions on the coordinate neighborhood $U$ in $J^{k}$. If $F \in C^{\infty}(M)$, then $\theta(F)$ on $U$ is

Received by the editors January 21, 1963 and, in revised form, May 25, 1963.

¿ Supported by ONR Contract 202736. 
the function $a^{\lambda}\left(\partial F / \partial y^{\lambda} \circ \beta\right)$. In these local coordinates the $i$ th prolongation of $\theta$ has the expression

$$
P^{i} \theta=a^{\lambda} \frac{\partial}{\partial y^{\lambda}}+\partial_{j}^{\#} a^{\lambda} \frac{\partial}{\partial p_{j}^{\lambda}}+\cdots+\partial_{j_{1}}^{\#} \cdots \partial_{j_{i}}^{\#} a^{\lambda} \frac{\partial}{\partial p_{j_{1}}^{\lambda} \cdots j_{i}} .
$$

(See [3, Lemma 1].) We shall also need the following lemma whose proof we omit.

Lemma 1. Let $\theta$ be a k-vector field, $F_{1}, F_{2} \in C^{\infty}\left(J^{i}\right), G \in C^{\infty}(M)$ and $F \in C^{\infty}\left(J^{i-j}\right)$, where $0<j<i$. Then

(A) $P^{i} \theta\left(F \circ \rho_{i-j}^{i}\right)=\left(P^{i-j} \theta(F)\right) \circ \rho_{i-j+k}^{i+k}$,

(B) $P^{i} \theta(G \circ \beta)=\theta(G) \circ \rho_{i}^{i+k}$,

(C) $P^{i} \theta\left(F_{1} F_{2}\right)=\left(F_{1} \circ \rho_{i}^{i+k}\right) P^{i} \theta\left(F_{2}\right)+\left(F_{2} \circ \rho_{i}^{i+k}\right) P^{i} \theta\left(F_{1}\right)$,

(D) $P^{i} \theta\left(\partial_{j_{1}}^{*} \cdots \partial_{j_{r}}^{\#} G \circ \rho_{r}^{i}\right)=\partial_{j_{1}}^{\#} \cdots \partial_{j_{r}}^{*} \theta(G) \circ \rho_{r+i}^{i+k}, r<k$,

(E) $P^{i} \theta\left(\partial_{j_{1}}^{\#} \cdots \partial_{j_{r}}^{*} F \circ \rho_{i-j+r}^{i}\right)=\left(\partial_{j_{1}}^{\#} \cdots \partial_{j_{r}}^{\#} P^{i-j \theta}(F)\right) \circ \rho_{i-j+k+r}^{i+k}, \quad r<j$. Conversely, if $\phi: C^{\infty}\left(J^{i}\right) \rightarrow C^{\infty}\left(J^{i+k}\right)$ satisfies $(\mathrm{A}), \cdots,(\mathrm{E})$ when $P^{i} \theta$ is replaced by $\phi$, then $\phi=P^{i} \theta$.

Another important property for us is that if $F \in C^{\infty}\left(J^{i}\right), f: N \rightarrow M$, then $\left(\partial / \partial x^{i}\right) G\left(j^{i}(f)\right)=\left(\partial_{i}^{*} G\right)\left(j^{i+1}(f)\right)$ for all $G \in C^{\infty}\left(J^{i}\right)$ [2, Proposition $1.10]$.

Let $I=(-\epsilon, \epsilon)$. An integral curve of $\theta$ starting at $f_{0}: N \rightarrow M$ is a 1-parameter family $f: N \times I \rightarrow M$ with $f_{0}(x)=f(x, 0)$ and for every $F \in C^{\infty}(M)$,

$$
\theta\left(j_{x}^{k}(f)\right)(F)=\theta(F)\left(j_{x}^{k}(f)\right)=\frac{\partial}{\partial t}(F \circ f)(x, t)=\left(\left(f * \frac{\partial}{\partial t}\right) F\right)(x, t) .
$$

2. Differential systems. A system $\Sigma$ of partial differential equations (s.p.d.e.) of order $h$ with $N$ as independent and $M$ as dependent variables is a finitely generated ideal in $C^{\infty}\left(J^{h}\right)$. A solution of $\Sigma$ is a map $f: N \rightarrow M$ such that $F\left(j_{x}^{h}(f)\right)=0$ for all $x \in N, F \in \Sigma$. $P^{k} \Sigma$ denotes the s.p.d.e. of order $h+k$ generated by the functions $F \circ \rho_{h}^{h+k}$, $\partial_{j}^{*} F \circ \rho_{h+1}^{h+k}, \cdots, \partial_{j_{1}}^{*} \cdots \partial_{j_{k}}^{*} F, 1 \leqq j, j_{t} \leqq n, F \in \Sigma$.

Definition. A $k$-vector field $\theta$ leaves $\Sigma$ invariant if for each $F \in \Sigma$, $P^{h} \theta(F) \in P^{k} \Sigma$.

Compare with [2] for the older theory. The intuitive meaning of invariance under a transformation group was that the transformations permute the solutions. We shall show that if $f_{0}$ is a solution of $\Sigma$ which belongs to an integral curve of $\theta$, then $\Sigma$ evaluated at this integral curve has zero derivatives at $f_{0}$ of all orders.

LEMma 2. If $\theta$ is an invariant vector field of $\Sigma$, then $\theta$ is an invariant vector field for $P^{i} \Sigma$, all $i$. 
This follows from (D) and (E) in Lemma 1. Using local coordinates, a calculation proves

Lemma 3. If $F \in C^{\infty}\left(J^{i}\right), f: N \times I \rightarrow M$, and $(\partial f / \partial t)=\theta\left(j_{x}^{k}(f)\right)$, then

$$
\frac{\partial}{\partial t} F\left(j_{x}^{i}(f)\right)=\left.P^{i} \theta(F)\right|_{j_{x}^{k+i}(f)}
$$

LemMa 4. If $f: N \rightarrow M$ is a solution of $\Sigma$, it is a solution of $P^{i} \Sigma$, all $i$.

Theorem 1. Suppose that

(A) $\theta$ is an invariant $k$-vector field of $\Sigma$,

(B) $f: N \times I \rightarrow M$ satisfies $(\partial f / \partial t)=\theta\left(j_{x}^{k}(f)\right)$, and

(C) $f(, 0): N \rightarrow M$ is a solution of $\Sigma$.

Then $\left.\left(\partial^{n} / \partial t^{n}\right) F\left(j_{x}^{h}(f)\right)\right|_{t=0}=0$ for all $x \in N, F \in \Sigma$, and $n=1,2, \cdots$.

Proof. From Lemma 3,

$$
\frac{\partial}{\partial t} F\left(j_{x}^{h}(f)\right)=\left.P^{h} \theta(F)\right|_{j x^{k+h}(f)} .
$$

However, $P^{h} \theta(F) \in P^{k} \Sigma$, and $f$ is a solution of $P^{k} \Sigma$ by Lemma 4 . Hence $\left.P^{h} \theta(F)\left(J_{x}^{k+h}(f)\right)\right|_{t=0}=0$, all $x \in N$. Let $F^{1}=P^{h} \theta(F) \in P^{k} \Sigma$. By Lemma 3,

$$
\left.P^{h+k} \theta(F)\right|_{j_{x}{ }^{2 k+h}(f)}=\frac{\partial}{\partial t} F^{1}\left(j_{x}^{h+k}(f)\right)=\frac{\partial}{\partial t}\left[\frac{\partial}{\partial t} F\left(j_{x}^{h}(f)\right)\right] .
$$

Using Lemma 4 as before, $\left.\left(\partial^{2} / \partial t^{2}\right) F\left(j_{x}^{h}(f)\right)\right|_{t=0}=0$. Continuing in this way, the result follows. Q.E.D.

When the manifolds and functions are real-analytic, Theorem 1 implies that integral curves of an invariant vector field which pass through one solution yield solutions for all parameter values.

\section{Lie algebra structure.}

Proposition. Let $\theta$ and $\psi$ be $k$ - and h-vector fields, respectively. Then

$$
P^{i}[\theta, \psi]=P^{i+h} \theta \circ P^{i} \psi-P^{i+k} \psi \circ P^{i} \theta .
$$

Proof. By induction on $i$. A local coordinate calculation shows the result for $i=1$. Call $\phi: C^{\infty}\left(J^{i}\right) \rightarrow C^{\infty}\left(J^{i+h+k}\right)$ the operator on the right-hand side. We shall use Lemma 1 . Let $F_{1}, F_{2} \in C^{\infty}\left(J^{i}\right), G \in C^{\infty}(M)$, and $F \in C^{\infty}\left(J^{i-j}\right)$.

$$
\begin{aligned}
P^{i+h} \theta \circ P^{i} \psi\left(F \circ \rho_{i-j}^{i}\right) & =P^{i+h} \theta\left(P^{i-j} \psi(F) \circ \rho_{i+h-j}^{i+h}\right) \\
& =\left(P^{i+h-j} \theta\left(P^{i-j} \psi(F)\right)\right) \circ \rho_{i+h+k-j}^{i+h+k} \\
& =\left(P^{i+h-j} \theta P^{i-j} \psi\right)(F) \circ \rho_{i+h+k-j}^{i+h+k}
\end{aligned}
$$


applying Lemma 1 (A) to $\psi$ and $\theta$. Interchanging $\theta$ and $\psi$, we find

$$
P^{i} \phi\left(F \circ \stackrel{i}{i-j}^{i}\right)=\left(P^{i-j} \phi(F)\right) \circ \stackrel{i+h+k}{\rho_{i+h+k-j}} .
$$

Now, by induction, $P^{i-i} \phi(F)=P^{i-j}[\theta, \psi]$. Hence (A) holds for $\phi$. The same technique works for (B), ․ (E). Q.E.D.

THEOREM 2. If $\theta$ and $\psi$ are $k$ - and h-vector fields, respectively, which leave $\Sigma$ invariant, then $[\theta, \psi]$ leaves $\Sigma$ invariant.

Proof. If $F \in \Sigma$ and $\Sigma$ is of order $i$, then $P^{i}[\theta, \psi](F)=P^{i+h} \theta \circ P^{i} \psi(F)$ $-P^{i+k} \psi \circ P^{i} \theta(F)$. However, $P \dot{\psi} \psi(F) \in P^{h} \Sigma$. By Lemma $2, \theta$ is an invariant vector field of $P^{h \Sigma}$, so $P^{i+h} \theta \circ P^{i} \psi(F) \in P^{h+k} \Sigma$. Similarly $P^{i+k} \psi \circ P^{i} \theta(F) \in P^{h+k} \Sigma$. Q.E.D.

We conclude that the set of all $k$-vector fields, $k=1,2, \cdots$, leaving $\Sigma$ invariant forms a Lie algebra under the bracket.

4. An example. Let $N=E^{n}, M=E^{m}$. Consider an s.p.d.e. of the type

$$
\frac{\partial y^{\lambda}}{\partial x^{n}}=\phi^{\lambda}\left(x^{1}, \cdots, x^{n-1}, y^{\mu}, \frac{\partial y^{\mu}}{\partial x^{1}}, \ldots, \frac{\partial y^{\mu}}{\partial x^{n-1}}\right),
$$

$\lambda, \mu=1, \cdots, m$. On $J^{1}$ let $F^{\lambda}=p_{n}^{\lambda}-\phi^{\lambda}\left(x^{i}, y^{\mu}, p_{i}^{\mu}\right)$, and let $\Sigma$ be generated by $F^{1}, \cdots, F^{m}$. Then by a calculation one may check that $\theta=\phi^{\lambda}\left(\partial / \partial y^{\lambda}\right)$ turns out to be an invariant vector field of $\Sigma$.

We can see that $\theta$ generates solutions of the Cauchy problem associated with $\Sigma$. Since $\theta$ is independent of $x^{n}$ and $p_{n}^{\lambda}$, it can be considered a 1-vector field on $E^{n-1}$. Suppose $f_{0}: E^{n-1} \rightarrow E^{m}$ is the initial data at $x^{n}=0$. Suppose $I=\left\{x^{n} \mid-\epsilon<x^{n}<\epsilon\right\}$ and $f: E^{n-1} \times I \rightarrow E^{m}$ is an integral curve of $\theta$ through $f_{0}$. But that is merely another way of saying that $f$ is a solution of $\Sigma$.

\section{REFERENCES}

1. R. Hermann, Formal tangency of vector fields in function spaces, Mimeographed Notes, Univ. of California, Berkeley, 1961.

2. H. H. Johnson, Classical differential invariants and applications to partial differential equations, Math. Ann. 148 (1962), 308-328.

3. - Bracket and exponential for a new type of vector field, Proc. Amer. Math. Soc. 15 (1964), 432-437.

4. M. Kuranishi, On E. Cartan's prolongation theorem of exterior differential systems, Amer. J. Math. 79 (1957), 1-47.

UNIVERSITY OF WASHINGTON 